\title{
Analysis of spatial vibrations of piezoceramic eccentric cylindrical shells interacting with an annular fluid layer
}

\author{
S.A. Bochkarev, S.V. Lekomtsev, A.N. Senin \\ Institute of Continuous Media Mechanics, Ural Branch Russian Academy of Sciences, Perm (Russian Federation) \\ bochkarev@icmm.ru, bttp:/ / orcid.org/0000-0002-9722-1269 \\ lekomtsev@icmm.ru,bttp://orcid.org/0000-0002-8331-2979 \\ senin.a@icmm.ru,bttps://orcid.org/0000-0002-7537-0001
}

\begin{abstract}
The work is devoted to a numerical study of the behavior of vertically oriented eccentric (non-coaxial) electroelastic shells, containing a fluid in the gap between them. The solution of the problem is found in the framework of a three-dimensional formulation using the finite element method. The shells are made of the piezoelectric material polarized in the radial direction. Thin-walled structures are considered in the framework of the classical theory based on the Kirchhoff - Love hypotheses, as well as the equations of linear electroelasticity. The distribution of the electric potential through the thickness is assumed linear. The fluid is considered within the framework of a potential theory. The study of the low natural frequencies and vibration modes is carried out for different variants of the boundary conditions at the edges of the shells, the level of fluid in the gap and the eccentricity of the inner shell. The influence of the electric boundary conditions specified on the parts of the shells surfaces covered with
\end{abstract}

\section{open 6 access}

Citation: Bochkarev, S.A., Lekomtsev, S.V., Senin A.N., Analysis of spatial vibrations of piezoceramic eccentric cylindrical shells interacting with an annular fluid laver, Frattura ed Integrità Strutturale, 49 (2019) 814-830.

Received: 01.04.2019

Accepted: 22.05.2019

Published: 01.07.2019

Copyright: (C) 2019 This is an open access article under the terms of the CC-BY 4.0, which permits unrestricted use, distribution, and reproduction in any medium, provided the original author and source are credited.

\section{INTRODUCTION}

I $\mathrm{n}$ the last few decades, the wide-spread use of structural elements in the form of nested shells with parallel axes, interacting with a fluid or gas contained in the gap between them, gave an impetus to the appearance of many publications, in which such systems are the subject of research. An extensive bibliography, covering recent studies into the dynamic behavior of coaxial shells interacting with both stationary and flowing fluids, is given in review [1] and monograph [2]. Smaller reviews extending this list of cited papers and including some unlisted pioneering studies are given in recent articles of the authors [3, 4]. However, in the context of this study, the matter of particular current interest is a few works that take into account the axis misalignment of the inner and outer shells [5-9]. In [5], it was shown that the 
axial eccentricity affects the vibration process only in the case of a narrow annular gap between infinite shells. The analysis of the added liquid masses for two infinite cylinders with eccentricity, containing either an ideal or a viscous fluid in the gap between them, was performed in [6] and [7]. As noted in [7], the added masses and viscous damping coefficients, which grow with increasing eccentricity, are of greater practical interest, because they are widely used in studying hydroelastic vibrations and stability of structures in various engineering applications. The analysis of natural frequencies of cylindrical shells with an eccentricity immersed in a rigid cylindrical container was performed in works [8,9]. The first of these papers considers the system, in which the non-viscous compressible fluid is contained only in the annular gap between the container and the shell, whereas the second paper examines the situation when the inner shell also contains a fluid. It has been demonstrated that with increasing eccentricity, the frequency of vibrations decreases, which is most significant for low circumferential and axial modes. Particular attention should also be paid to publications dealing with the analysis of coaxial shells partially filled with a fluid [10-15]. In all these papers, the studies of the dynamic behavior of coaxial shells are made with account of the free-surface sloshing effect, but do not include numerical simulation, which would allow one to estimate the effect of this boundary condition. Note that here we cite only those works which analyze the effect of partial filling of shells on the dynamic characteristics of the system. It is shown that an increase in the fluid level causes a decrease in the natural vibration frequencies. A more general review of papers aimed at studying hydroelastic vibrations of partially filled coaxial cylindrical shells is given in monograph [16].

In the above mentioned works, the solution of the problem is searched for in the context of a two-dimensional (flat) or axisymmetric problem formulation. In the case when non-coaxial structures are set in the vertical or horizontal position and partial filled with fluid, their symmetry with respect to the circumferential coordinate is disturbed, which necessitates the use of more complex spatial models. As far as the authors know, such studies, including those that take into account the elasticity of both shells, are not presented in the literature.

Electroelastic materials embedded in or attached to engineering structures have been used for rather long time in various fields of technology in order to improve the performance characteristics of a product. Intellectual systems of passive or active control of dynamic behavior built on the basis of their peculiar properties serve to reduce the level of mechanical vibrations or acoustic noise. The bibliography of basic works dealing with the analysis of various approaches used in modeling the structures with piezoelectric elements, and presenting examples of their practical use can be found in monograph [17]. In [18], the authors come to a conclusion that successful practical application of piezoelectric elements coming in contact with a fluid or gaseous medium is possible only with an adequate description of their coupled response. In the case of thin-walled structures this can be achieved with the model proposed in [19]. The model is based on a singlelayer representation of the elastic body displacements and a layered description of piezoelectric properties. This approach was used for modeling the plates and single shells, including those interacting with a quiescent fluid, and also the elastic structures with external patches made completely or partially from the electroelastic material [20-23]. For a linear distribution of electrical properties through the body thickness, this method, when used in the framework of finite element modeling, allows us to simplify the solution by eliminating the electrical components at the element level. In the literature, there are no papers describing the application of the above method to coaxial shells.

Thus, the purpose of this work is to analyze the natural frequencies and the corresponding modes of vibrations of elastic and piezoelastic eccentric (non-coaxial) cylindrical shells at different values of the annular gap width and different levels of ideal compressible fluid contained in this gap. The solution of the problem is found using a modified version of the finite element algorithm developed in the previous works for evaluating the hydroelastic interaction of single shell structures with arbitrary cross section completely or partially filled with fluid [24-26].

\section{STATEMENT OF THE PROBLEM AND CONSTITUTIVE RELATIONS}

$\mathrm{W}$ e consider a structure consisting of two vertically located eccentric (non-coaxial) shells of length $L$, radiuses $R^{(1)}$ and $R^{(2)}$, and thicknesses $b^{(1)}$ and $b^{(2)}$ (Fig. 1) made of electroelastic material (piezoceramics). Hereinafter, the superscripts "(1)" and "(2)" characterize the inner and outer shells, respectively. The axis of rotation of the inner shell is displaced laterally with respect to rotation axis of the outer shell by the value $a$ $\left(|a|<\mathrm{R}^{(2)}-\mathrm{R}^{(1)}\right)$, and the space between them is filled with an ideal compressible fluid to the level $H(0 \leq H \leq L)$. It is necessary to investigate the natural frequencies and modes of vibrations of the system under different kinematic and electric boundary conditions, and geometric parameters (the size of the annular gap, the height of the fluid, eccentricity). 


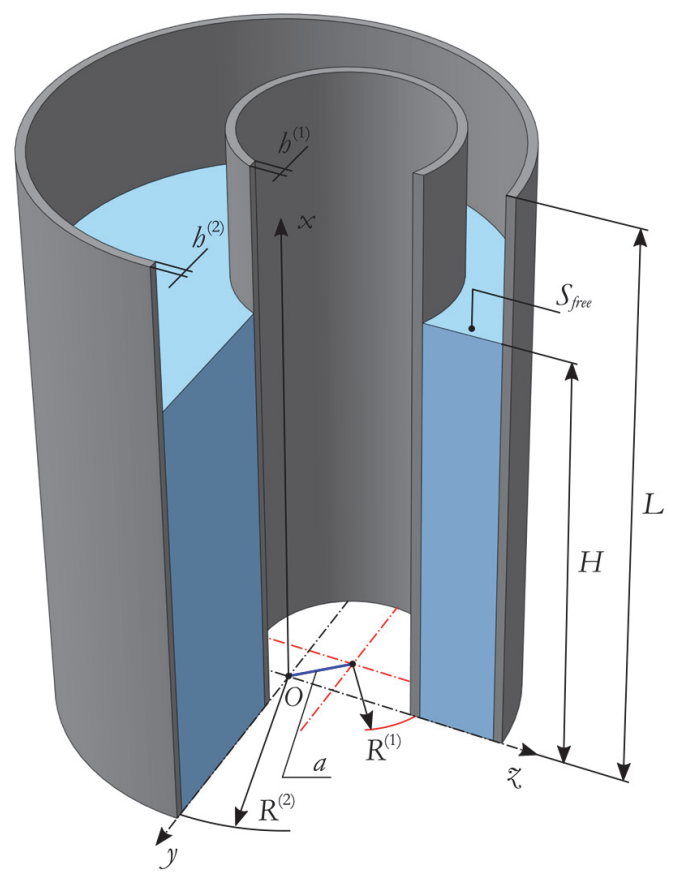

Figure 1: Schematic representation of the computational domain.

An ideal compressible fluid is considered in the framework of a potential theory, in which the wave equation in the case of small perturbations takes the following form [27]:

$$
\nabla^{2} \phi=\frac{1}{c^{2}} \frac{\partial \phi}{\partial t}
$$

where $\phi$ is the velocity potential, $c$ is the speed of sound in the liquid, $\nabla$ is the differential Nabla operator, $\partial / \partial t$ is the time derivative. Using the perturbation velocity potential as an unknown function allows to take into account both the flow or the rotation of the inviscid compressible fluid with a slight modification of the numerical algorithm.

In the case of complete filling of the shell with a fluid, the following boundary conditions are prescribed for the velocity potential:

$$
x=0, L: \quad \partial \phi / \partial x=0
$$

In the case of partial filling, it is assumed that the free surface of the fluid $S_{\text {free }}$ remains stationary and is free from the dynamic pressure and surface tension. The corresponding boundary condition is given as [28]

$$
x=H: \quad \phi=0
$$

On the wetted surfaces $S_{\sigma}^{(i)}=S_{f} \cap S_{s}^{(i)}$ the impermeability conditions hold true

$$
\frac{\partial \phi}{\partial \mathbf{n}^{(i)}}= \pm \frac{\partial w^{(i)}}{\partial t}
$$

where $\mathbf{n}^{(i)}$ is the vector of the normal to the shell surface; $w^{(i)}$ is the normal component of shell displacements; $S_{f}$ and $S_{s}^{(i)}$ are the surfaces that bound the volumes of the fluid $V_{f}$ and shell $V_{s}^{(i)}$. In what follows, $i=\overline{1,2}$. The hydrodynamic pressure $p$ exerted on the elastic structures by the fluid is calculated using the Bernoulli equation: 


$$
p^{(i)}=\mp \rho_{f} \frac{\partial \phi}{\partial t}
$$

where $\rho_{f}$ is the fluid density and the sign of the right-hand parts of Eqns. (3)-(4) depends on the direction of the vectors of the normal to the external surfaces of the shells.

Eqn. (1) together with the boundary conditions (2)-(3) are converted by the Bubnov - Galerkin method to a weak form $[26]$

$$
\int_{V_{f}} \nabla F_{n} \nabla \hat{\phi} \mathrm{d} V+\int_{V_{f}} F_{n} \frac{1}{c^{2}} \frac{\partial^{2} \hat{\phi}}{\partial t^{2}} \mathrm{~d} V-\int_{S_{\sigma}^{(1)}} F_{n} \frac{\partial \hat{w}^{(1)}}{\partial t} \mathrm{~d} S+\int_{S_{\sigma}^{(2)}} F_{n} \frac{\partial \hat{w}^{(2)}}{\partial t} \mathrm{~d} S=0, \quad n=\overline{1, m_{f}}
$$

where $\hat{\phi}$ and $\hat{w}^{(i)}$ are the approximations of the velocity potential and the normal components of the vectors of shell displacements; $F_{n}$ and $m_{f}$ are the basis functions and their number, respectively.

In the general case, the behavior of the electroelastic body is described by the equations of state, piezoelectric effect, and the electrostatic relations $[29,30]$

$$
\begin{aligned}
& \boldsymbol{\sigma}=\mathbf{c} \boldsymbol{\varepsilon}-\mathbf{e}^{\mathrm{T}} \mathbf{E}, \\
& \mathbf{D}=\mathbf{e} \boldsymbol{\varepsilon}+\mathbf{d E}, \\
& \nabla \cdot \mathbf{D}=0, \\
& \mathbf{E}=-\nabla \psi,
\end{aligned}
$$

Here: $\sigma, \varepsilon$ are the stress and linear strain tensors; $\mathbf{E}$ and $\mathbf{D}$ are the vectors of electric field intensity and electrical induction; c, e, $\mathbf{d}$ are the matrices of elastic constants, piezoelectric and dielectric coefficients; $\psi$ is the electrostatic potential. In the case when a thin-walled structure with a radially polarized field is in the plane stress state, Eqns. (6)-(9) can be simplified. In particular, of all components the vectors of the electric field strength and nonzero electrical induction retain only $E_{3}$ and $D_{3}$, and Eqns. (6) and (7) are written as follows [31, 32]:

$$
\begin{aligned}
& \left\{\begin{array}{c}
\sigma_{11} \\
\sigma_{22} \\
\tau_{12}
\end{array}\right\}=\tilde{\mathbf{c}}\left\{\begin{array}{l}
\varepsilon_{11} \\
\varepsilon_{22} \\
\gamma_{12}
\end{array}\right\}+\left[\begin{array}{ccc}
0 & 0 & \tilde{e}_{31} \\
0 & 0 & \tilde{e}_{32} \\
0 & 0 & 0
\end{array}\right]\left\{\begin{array}{c}
0 \\
0 \\
E_{3}
\end{array}\right\}, \quad \tilde{\mathbf{c}}=\left[\begin{array}{ccc}
\tilde{c}_{11} & \tilde{c}_{12} & 0 \\
\tilde{c}_{21} & \tilde{c}_{22} & 0 \\
0 & 0 & \tilde{c}_{66}
\end{array}\right], \\
& \left\{\begin{array}{c}
0 \\
0 \\
D_{3}
\end{array}\right\}=\left[\begin{array}{ccc}
0 & 0 & 0 \\
0 & 0 & 0 \\
\tilde{e}_{31} & \tilde{e}_{32} & 0
\end{array}\right]\left\{\begin{array}{l}
\varepsilon_{11} \\
\varepsilon_{22} \\
\gamma_{12}
\end{array}\right\}+\left[\begin{array}{ccc}
0 & 0 & 0 \\
0 & 0 & 0 \\
0 & 0 & \tilde{d}_{33}
\end{array}\right]\left\{\begin{array}{c}
0 \\
0 \\
E_{3}
\end{array}\right\},
\end{aligned}
$$

where

$$
\tilde{c}_{l m}=c_{l m}-c_{l 3} c_{m 3} / c_{33}, \quad \tilde{c}_{66}=c_{66}, \quad \tilde{e}_{3 m}=e_{3 m}-e_{33} c_{m 3} / c_{33}, \quad \tilde{d}_{33}=d_{33}+e_{33}^{2} / c_{33}, \quad l, m=\overline{1,2}
$$

Here we proceed from the assumption that the shell surface is covered with thin weightless electrodes, which can either be shorted, which corresponds to the boundary condition $E_{3}=0$ (short circuited) or disconnected $\left(D_{3}=0\right.$, open circuited). From Eqns. (6)-(9) the following integral relation can be obtained [21] 


$$
\int_{V_{s}}(\delta \mathbf{E})^{\mathrm{T}} \mathbf{e} \varepsilon \mathrm{d} V+\int_{V_{s}}(\delta \mathbf{E})^{\mathrm{T}} \mathbf{d} \mathbf{E} \mathrm{d} V=0
$$

In the shell simulation, it is assumed that their curved surfaces can be represented with a reasonable degree of accuracy as a set of flat segments [33]. The strains in each segment are determined according to the classical theory of thin plates [34]. The corresponding relations in the Cartesian coordinates $(\bar{x}, \bar{y}, \bar{z})$, which are tied in with the lateral surfaces of thinwalled structures, are written as

$$
\begin{aligned}
& \overline{\boldsymbol{\varepsilon}}^{(i)}=\left\{\overline{\boldsymbol{\varepsilon}}_{11}^{(i)}, \bar{\varepsilon}_{22}^{(i)}, \bar{\gamma}_{12}^{(i)}\right\}^{\mathrm{T}}=\tilde{\boldsymbol{\varepsilon}}^{(i)}+\bar{z} \boldsymbol{\kappa}^{(i)}, \\
& \tilde{\boldsymbol{\varepsilon}}^{(i)}=\left\{\tilde{\varepsilon}_{11}^{(i)}, \tilde{\varepsilon}_{22}^{(i)}, \tilde{\gamma}_{12}^{(i)}\right\}^{\mathrm{T}}=\left\{\frac{\partial u^{(i)}}{\partial \bar{x}}, \frac{\partial v^{(i)}}{\partial \bar{y}}, \frac{\partial u^{(i)}}{\partial \bar{y}}+\frac{\partial v^{(i)}}{\partial \bar{x}}\right\}^{\mathrm{T}}, \\
& \boldsymbol{\kappa}^{(i)}=\left\{\overline{\boldsymbol{\kappa}}_{11}^{(i)}, \overline{\boldsymbol{\kappa}}_{22}^{(i)}, \overline{\boldsymbol{\kappa}}_{12}^{(i)}\right\}^{\mathrm{T}}=\left\{-\frac{\partial^{2} w^{(i)}}{\partial \bar{x}^{2}},-\frac{\partial^{2} w^{(i)}}{\partial \bar{y}^{2}},-2 \frac{\partial^{2} w^{(i)}}{\partial \bar{x} \partial \bar{y}}\right\}^{\mathrm{T}} .
\end{aligned}
$$

Hereinafter, the over-line means that the quantity is defined in the coordinates $(\bar{x}, \bar{y}, \bar{z})$, and $\bar{u}^{(i)}, \bar{v}^{(i)}$ and $\bar{w}^{(i)}$ is the meridional, circumferential and normal components of the displacement vectors of the middle surfaces of the shells in these coordinates.

Taking into account the accepted simplifications, the physical relations interconnecting the vectors of generalized forces and moments $\overline{\mathbf{T}}^{(i)}$, generalized deformations $\overline{\boldsymbol{\varepsilon}}^{(i)}$ and electric field strength $\mathbf{E}^{(i)}$, can be written in the matrix form as

$$
\begin{aligned}
& \overline{\mathbf{T}}^{(i)}=\tilde{\mathbf{D}}^{(i)} \overline{\boldsymbol{\varepsilon}}^{(i)}-\mathbf{G}^{(i)} \mathbf{E}^{(i)}, \quad \overline{\mathbf{T}}^{(i)}=\left\{\bar{T}_{11}^{(i)}, \bar{T}_{22}^{(i)}, \bar{T}_{12}^{(i)}, \bar{M}_{11}^{(i)}, \bar{M}_{22}^{(i)}, \bar{M}_{12}^{(i)}\right\}^{\mathrm{T}}, \\
& \tilde{\mathbf{D}}^{(i)}=\left[\begin{array}{ll}
\tilde{\mathbf{A}}^{(i)} & \tilde{\mathbf{B}}^{(i)} \\
\tilde{\mathbf{B}}^{(i)} & \tilde{\mathbf{C}}^{(i)}
\end{array}\right], \quad \overline{\boldsymbol{\varepsilon}}^{(i)}=\left\{\overline{\boldsymbol{\varepsilon}}_{11}^{(i)}, \bar{\varepsilon}_{22}^{(i)}, \bar{\gamma}_{12}^{(i)}, \overline{\boldsymbol{\kappa}}_{11}^{(i)}, \overline{\boldsymbol{\kappa}}_{22}^{(i)}, \overline{\boldsymbol{\kappa}}_{12}^{(i)}\right\}^{\mathrm{T}},
\end{aligned}
$$

where the coefficients entering into the stiffness matrix $\tilde{\mathbf{D}}^{(i)}$ are calculated as

$$
\left(\tilde{\mathbf{A}}^{(i)}, \tilde{\mathbf{B}}^{(i)}, \tilde{\mathbf{C}}^{(i)}\right)=\int_{-b^{(i)} / 2}^{b^{(i)} / 2}\left(1, \bar{z}, \bar{z}^{2}\right) \tilde{\mathbf{c}}^{(i)} \mathrm{d} \bar{z},
$$

and the structure of the matrix $\mathbf{G}^{(i)}$ will be presented below.

A mathematical formulation of the problem of the dynamics of elastic shells is based on the variational principle of virtual displacements, which, with account of relation (4) and the work made by the inertial forces, can be written in the matrix form as

$$
\begin{aligned}
& \int_{V_{s}^{(i)}}\left(\delta \overline{\boldsymbol{\varepsilon}}^{(i)}\right)^{\mathrm{T}} \overline{\mathbf{T}}^{(i)} d V+\int_{V_{s}^{(i)}} \rho_{s}^{(i)}\left(\delta \overline{\mathbf{u}}^{(i)}\right)^{\mathrm{T}} \ddot{\ddot{\mathbf{u}}^{(i)}} d V-\int_{S_{\sigma}^{(i)}}\left(\delta \overline{\mathbf{u}}^{(i)}\right)^{\mathrm{T}} \overline{\mathbf{P}}^{(i)} d S=\mathbf{0}, \\
& \overline{\mathbf{u}}^{(i)}=\left\{u^{(i)}, v^{(i)}, w^{(i)}, \theta_{\bar{x}}^{(i)}, \theta_{\bar{y}}^{(i)}, \theta_{\bar{z}}^{(i)}\right\}^{\mathrm{T}}, \quad \overline{\mathbf{P}}^{(i)}=\left\{0,0, p^{(i)}, 0,0,0\right\}^{\mathrm{T}},
\end{aligned}
$$

where $\rho_{s}^{(i)}$ are the densities of shell materials, $\overline{\mathbf{u}}^{(i)}$ are the vectors of the displacements and rotation angles of shells, and $\overline{\mathbf{P}}^{(i)}$ are the vectors of loads acting on the shell surfaces. 


\section{NUMERICAL IMPLEMENTATION}

$\mathrm{F}$

ollowing [21], let us decompose the shells through its thickness into $N$ layers and represent the component of the electric field $E_{3}$ for each layer as

$$
\left(E_{3}\right)_{k}^{(i)}=-V_{k}^{(i)} / b_{k}^{(i)},
$$

where $b_{k}^{(i)}=\bar{z}_{k}^{(i)}-\bar{z}_{k-1}^{(i)}$ is the thickness of the $k$-th layer; $\bar{z}^{(i)}\left(-b^{(i)} / 2 \leq \bar{z}^{(i)} \leq b^{(i)} / 2\right)$ is the coordinate measured from the middle surface of the shell; $V_{k}^{(i)}=\psi_{k}^{(i)}-\psi_{k-1}^{(i)}$ is the difference in the electrostatic potentials between the upper and lower surfaces of the layer, which, as well as the components of the vectors of shell displacements $\mathbf{u}^{(i)}$ and the velocity potential $\phi$, turns to be an unknown variable.

The numerical solution of the problem is found using the finite element method (FEM) [33]. To describe the velocity potential $\hat{\phi}$, the basic functions $F_{n}$, and membrane displacements of shells $\left(u^{(i)}, v^{(i)}\right)$ we use the Lagrange shape functions with linear approximation, and for bending deflections of shells $w^{(i)}$ we use the Hermite non-conformal shape functions The discretization of the fluid and shell regions is made using spatial prismatic (8-node brick) and flat quadrangular (4-node plane) finite elements, respectively.

Expressing the unknown variables in terms of their nodal values, we arrive at the following matrix relations

$$
\overline{\mathbf{u}}^{(i)}=\mathbf{N}^{(i)} \overline{\mathbf{u}}_{e}^{(i)}, \quad \phi=\mathbf{F} \phi_{e}, \quad \overline{\boldsymbol{\varepsilon}}^{(i)}=\mathbf{B}^{(i)} \overline{\mathbf{u}}_{e}^{(i)}, \quad \mathbf{E}_{e}^{(i)}=-\mathbf{B}_{\psi}^{(i)} \mathbf{\Phi}_{e}^{(i)},
$$

where $\mathbf{F}$ and $\mathbf{N}^{(i)}$ are the shape functions for the velocity potential of the fluid and the vectors of the nodal displacements of the shell, $\overline{\mathbf{u}}_{e}^{(i)}$ and $\boldsymbol{\phi}_{e}$ are the vectors of the nodal values, $\mathbf{B}^{(i)}$ are the gradient matrices which are determined by Eqn. (14) and relate the strain vectors to the shell displacements, $\mathbf{E}_{e}^{(i)}=\left\{E_{1}^{(i)}, \ldots E_{k}^{(i)}, \ldots E_{N}^{(i)}\right\}^{\mathrm{T}}$, $\boldsymbol{\Phi}_{e}^{(i)}=\left\{V_{1}^{(i)}, \ldots V_{k}^{(i)}, \ldots V_{N}^{(i)}\right\}^{\mathrm{T}}, \mathbf{B}_{\psi}^{(i)}=\operatorname{diag}\left\{1 / h_{1}^{(i)}, \ldots, 1 / h_{k}^{(i)}, \ldots 1 / h_{N}^{(i)}\right\}$. Using the introduced relations the matrix $\mathbf{G}^{(i)}$ in Eqn. (15) is generated in the following way

$$
\mathbf{G}^{(i)}=\left[\begin{array}{ccccc}
\hat{G}_{11}^{(i)} & \ldots & \hat{G}_{1 k}^{(i)} & \ldots & \hat{G}_{1 N}^{(i)} \\
\hat{G}_{21}^{(i)} & \ldots & \hat{G}_{2 k}^{(i)} & \ldots & \hat{G}_{2 N}^{(i)} \\
0 & \ldots & 0 & \ldots & 0 \\
\tilde{G}_{11}^{(i)} & \ldots & \tilde{G}_{1 k}^{(i)} & \ldots & \tilde{G}_{1 N}^{(i)} \\
\tilde{G}_{21}^{(i)} & \ldots & \tilde{G}_{2 k}^{(i)} & \ldots & \tilde{G}_{2 N}^{(i)} \\
0 & \ldots & 0 & \ldots & 0
\end{array}\right],
$$

where

$$
\hat{G}_{l k}^{(i)}=b_{k}^{(i)}\left(\tilde{e}_{3 l}^{(i)}\right)_{k}, \quad \tilde{G}_{l k}^{(i)}=\frac{1}{2}\left[\left(z_{k}^{(i)}\right)^{2}-\left(z_{k-1}^{(i)}\right)^{2}\right]\left(\tilde{e}_{3 l}^{(i)}\right)_{k}, \quad k=\overline{1, N}, \quad l=\overline{1,2} .
$$

From Eqns. (5), (13) and (17) taking into account (18)-(20), we derive with the aid of the standard FEM operations a coupled system of equations, which can be used to describe the interaction of electroelastic shells with an ideal compressible fluid. The system can be represented in a matrix form as 


$$
\begin{aligned}
& {\left[\begin{array}{ccccc}
\mathbf{M}_{s}^{(1)} & 0 & 0 & 0 & 0 \\
0 & 0 & 0 & 0 & 0 \\
0 & 0 & \mathbf{M}_{s}^{(2)} & 0 & 0 \\
0 & 0 & 0 & 0 & 0 \\
0 & 0 & 0 & 0 & \mathbf{M}_{f}
\end{array}\right]\left\{\begin{array}{c}
\ddot{\mathbf{u}}^{(1)} \\
\ddot{\boldsymbol{\psi}}^{(1)} \\
\ddot{\mathbf{u}}^{(2)} \\
\ddot{\boldsymbol{\psi}}^{(2)} \\
\ddot{\boldsymbol{\phi}}
\end{array}\right\}+\left[\begin{array}{ccccc}
0 & 0 & 0 & 0 & -\mathbf{C}_{s f}^{(1)} \\
0 & 0 & 0 & 0 & 0 \\
0 & 0 & 0 & 0 & \mathbf{C}_{s f}^{(2)} \\
0 & 0 & 0 & 0 & 0 \\
-\mathbf{C}_{f s}^{(1)} & 0 & \mathbf{C}_{f s}^{(2)} & 0 & 0
\end{array}\right]\left\{\begin{array}{c}
\dot{\mathbf{u}}^{(1)} \\
\dot{\boldsymbol{\psi}}^{(1)} \\
\dot{\mathbf{u}}^{(2)} \\
\dot{\boldsymbol{\psi}}^{(2)} \\
\dot{\boldsymbol{\phi}}
\end{array}\right\}+} \\
& +\left[\begin{array}{ccccc}
\mathbf{K}_{s}^{(1)} & -\mathbf{K}_{\psi}^{(1)} & 0 & 0 & 0 \\
\left(\mathbf{K}_{s \psi}^{(1)}\right)^{\mathrm{T}} & \mathbf{K}_{\psi}^{(1)} & 0 & 0 & 0 \\
0 & 0 & \mathbf{K}_{s}^{(2)} & -\mathbf{K}_{s \psi}^{(2)} & 0 \\
0 & 0 & \left(\mathbf{K}_{s \psi}^{(1)}\right)^{\mathrm{T}} & \mathbf{K}_{\psi}^{(2)} & 0 \\
0 & 0 & 0 & 0 & \mathbf{K}_{f}
\end{array}\right]\left\{\begin{array}{c}
\mathbf{u}^{(1)} \\
\boldsymbol{\psi}^{(1)} \\
\mathbf{u}^{(2)} \\
\boldsymbol{\psi}^{(2)} \\
\boldsymbol{\phi}
\end{array}\right\}=0,
\end{aligned}
$$

where $\mathbf{u}^{(i)}, \phi$ and $\psi^{(i)}$ are the generalized vectors of the shell displacements and rotation angles, the perturbation velocity potential of the fluid and the difference in the electrostatic potentials of the shells.

The typical stiffness $(\mathbf{K})$, mass $(\mathbf{M})$ and damping $(\mathbf{C})$ matrices for individual finite elements are generated as follows:

$$
\begin{aligned}
& \mathbf{K}_{f}=\int_{V_{f}}\left(\frac{\partial \mathbf{F}^{\mathrm{T}}}{\partial x} \frac{\partial \mathbf{F}}{\partial x}+\frac{\partial \mathbf{F}^{\mathrm{T}}}{\partial y} \frac{\partial \mathbf{F}}{\partial y}+\frac{\partial \mathbf{F}^{\mathrm{T}}}{\partial z} \frac{\partial \mathbf{F}}{\partial z}\right) \mathrm{d} V, \quad \mathbf{M}_{f}=\int_{V_{f}} \frac{1}{c^{2}} \mathbf{F}^{\mathrm{T}} \mathbf{F} \mathrm{d} V, \quad \overline{\mathbf{C}}_{f s}^{(i)}=-\int_{S_{\sigma}^{(i)}} \mathbf{F}^{\mathrm{T}} \mathbf{N}_{w}^{(i)} \mathrm{d} S, \\
& \overline{\mathbf{K}}_{s}^{(i)}=\int_{V_{s}^{(i)}}\left(\mathbf{B}^{(i)}\right)^{\mathrm{T}} \tilde{\mathbf{D}}^{(i)} \mathbf{B}^{(i)} \mathrm{d} V, \quad \overline{\mathbf{M}}_{s}^{(i)}=\int_{V_{s}^{(i)}} \rho_{s}^{(i)}\left(\mathbf{N}^{(i)}\right)^{\mathrm{T}} \mathbf{N}^{(i)} \mathrm{d} V, \quad \overline{\mathbf{C}}_{s f}^{(i)}=\int_{S_{\sigma}^{(i)}} \rho_{f}\left(\mathbf{N}_{w}^{(i)}\right)^{\mathrm{T}} \mathbf{F} \mathrm{d} S, \\
& \overline{\mathbf{K}}_{s \psi}^{(i)}=\int_{V_{s}^{(i)}}\left(\mathbf{B}^{(i)}\right)^{\mathrm{T}} \mathbf{G}^{(i)} \mathbf{B}_{\psi}^{(i)} \mathrm{d} V, \quad \mathbf{K}_{\psi}^{(i)}=\int_{V_{s}^{(i)}}\left(\mathbf{B}_{\psi}^{(i)}\right)^{\mathrm{T}} \mathbf{H}^{(i)} \mathbf{B}_{\psi}^{(i)} \mathrm{d} V, \\
& \mathbf{H}^{(i)}=\operatorname{diag}\left\{h_{1}^{(i)}\left(\tilde{d}_{33}^{(i)}\right)_{1}, \ldots, h_{k}^{(i)}\left(\tilde{d}_{33}^{(i)}\right)_{k}, \ldots, h_{N}^{(i)}\left(\tilde{d}_{33}^{(i)}\right)_{N}\right\} .
\end{aligned}
$$

Here $\mathbf{N}_{w}^{(i)}$ are the shape functions for the normal component of the nodal displacement vector of the shells.

Since some matrices in (23) are written in the Cartesian coordinates $(\bar{x}, \bar{y}, \bar{z})$, the derivation of the solution in the global coordinates, which have been used to specify the problem geometry and to write the constitutive equations for the fluid, involves the transformation of the displacements and rotation angles using the matrix of the directional cosines $\gamma$ of size $3 \times 3$ in the following way:

$$
\mathbf{u}^{(i)}=\left[\begin{array}{ll}
\boldsymbol{\gamma} & 0 \\
0 & \boldsymbol{\gamma}
\end{array}\right] \overline{\mathbf{u}}^{(i)} .
$$

During the numerical implementation, at each node of the finite element of the shell it is necessary to determine six unknowns (three displacements and three angles of rotation). Therefore, the transformation of conventional matrices is performed using the diagonal matrix $\mathbf{L}=\operatorname{diag}\{\boldsymbol{\gamma}, \boldsymbol{\gamma}, \boldsymbol{\gamma}, \boldsymbol{\gamma}, \boldsymbol{\gamma}, \boldsymbol{\gamma}, \boldsymbol{\gamma}, \boldsymbol{\gamma}\}$ :

$$
\begin{aligned}
& \mathbf{K}_{s}^{(i)}=\left(\mathbf{L}^{(i)}\right)^{\mathrm{T}} \overline{\mathbf{K}}_{s}^{(i)} \mathbf{L}^{(i)}, \quad \mathbf{M}_{s}^{(i)}=\left(\mathbf{L}^{(i)}\right)^{\mathrm{T}} \overline{\mathbf{M}}_{s}^{(i)} \mathbf{L}^{(i)}, \quad \mathbf{C}_{s f}^{(i)}=\left(\mathbf{L}^{(i)}\right)^{\mathrm{T}} \overline{\mathbf{C}}_{s f}^{(i)}, \\
& \mathbf{K}_{s \psi}^{(i)}=\left(\mathbf{L}^{(i)}\right)^{\mathrm{T}} \overline{\mathbf{K}}_{s \psi}^{(i)}, \quad \mathbf{C}_{f s}^{(i)}=\overline{\mathbf{C}}_{f s}^{(i)} \mathbf{L}^{(i)} .
\end{aligned}
$$

Expressing the quantities associated with the electrostatic potential in terms of the shell displacements 


$$
\boldsymbol{\psi}^{(i)}=-\left(\mathbf{K}_{\psi}^{(i)}\right)^{-1}\left(\mathbf{K}_{s \psi}^{(i)}\right)^{\mathrm{T}} \mathbf{u}^{(i)}
$$

yields, instead of (22), the system of equations with respect to the displacements of electroelastic bodies and the velocity potential of the fluid

$$
\begin{aligned}
& {\left[\begin{array}{ccc}
\mathbf{M}_{s}^{(1)} & 0 & 0 \\
0 & \mathbf{M}_{s}^{(2)} & 0 \\
0 & 0 & \mathbf{M}_{f}
\end{array}\right]\left\{\begin{array}{c}
\ddot{\mathbf{u}}^{(1)} \\
\ddot{\mathbf{u}}^{(2)} \\
\ddot{\boldsymbol{\phi}}
\end{array}\right\}+\left[\begin{array}{ccc}
0 & 0 & -\mathbf{C}_{s f}^{(1)} \\
0 & 0 & \mathbf{C}_{s f}^{(2)} \\
-\mathbf{C}_{f s}^{(1)} & \mathbf{C}_{f s}^{(2)} & 0
\end{array}\right]\left\{\begin{array}{l}
\dot{\mathbf{u}}^{(1)} \\
\dot{\mathbf{u}}^{(2)} \\
\dot{\boldsymbol{\phi}}
\end{array}\right\}+} \\
& +\left[\begin{array}{ccc}
\mathbf{K}_{s}^{(1)}+\mathbf{K}_{s \psi}^{(1)}\left(\mathbf{K}_{\psi}^{(1)}\right)^{-1}\left(\mathbf{K}_{s \psi}^{(1)}\right)^{\mathrm{T}} & 0 & 0 \\
0 & \mathbf{K}_{s}^{(2)}+\mathbf{K}_{s \psi}^{(2)}\left(\mathbf{K}_{\psi}^{(2)}\right)^{-1}\left(\mathbf{K}_{s \psi}^{(2)}\right)^{\mathrm{T}} & 0 \\
0 & 0 & \mathbf{K}_{f}
\end{array}\right]\left\{\begin{array}{c}
\mathbf{u}^{(1)} \\
\mathbf{u}^{(2)} \\
\boldsymbol{\phi}
\end{array}\right\}=0
\end{aligned}
$$

which can be written in a more compact form

$$
\mathbf{M} \ddot{\mathbf{x}}+\mathbf{C} \dot{\mathbf{x}}+\mathbf{K} \mathbf{x}=0, \quad \mathbf{x}=\left\{\mathbf{u}^{(1)}, \mathbf{u}^{(2)}, \boldsymbol{\phi}\right\}^{\mathrm{T}}
$$

Representing the motion of shells and fluid in the exponential form

$$
\left(\mathbf{u}^{(i)}, \boldsymbol{\phi}\right)=\left(\tilde{\mathbf{u}}^{(i)}(x, y, z), \tilde{\phi}(x, y, z)\right) \exp (\lambda t)
$$

we obtain instead of (28) the following expression

$$
\lambda^{2} \mathbf{M} \mathbf{x}+\lambda \mathbf{C x}+\mathbf{K} \mathbf{x}=0
$$

Here, $\tilde{\mathbf{u}}^{(i)}$ and $\tilde{\boldsymbol{\phi}}^{(i)}$ are some functions of the coordinates; $\lambda=\delta+\mathrm{i} \omega$ is the characteristic coefficient, in which $\omega$ is the natural frequency of vibrations, $\delta$ is the quantity responsible for the system damping, and $\mathrm{i}=\sqrt{-1}$ is the imaginary unit. The system of Eqns. (30) is reduced to an asymmetric generalized eigenvalue problem

$$
\left(\left[\begin{array}{cc}
\mathbf{C} & \mathbf{K} \\
-\mathbf{I} & 0
\end{array}\right]+\lambda\left[\begin{array}{cc}
\mathbf{M} & 0 \\
0 & \mathbf{I}
\end{array}\right]\right)\left\{\begin{array}{c}
\lambda \mathbf{x} \\
\mathbf{x}
\end{array}\right\}=0
$$

where $\mathbf{I}$ is the unit matrix.

The calculation of the eigenvalues of the system of Eqns. (31) is carried out using the ARPACK procedures, which are based on the implicitly restarted Arnoldi method [35].

\section{RESULTS}

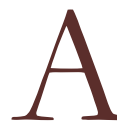

few numerical examples were considered to investigated the behavior of vertically oriented coaxial cylindrical shells, containing an ideal compressible fluid in the annular gap between them. The system parameters are listed in Tab. 1. The shells were made of PZT-5H piezoceramics, the physical and mechanical characteristics of which are given in Tab.2. As the boundary conditions for thin-walled bodies, we chose rigid clamping $\left(u=v=w=\theta_{x}=\theta_{y}=\theta_{z}=0\right)$ at one end (cantilever, CF) or at both ends (clamped-clamped, CC). The calculations were 
performed at different values of the dimensionless annular gap $k$, filling level $\eta$ and displacement (eccentricity) of the inner shell $\xi$

$$
k=\left(R^{(2)}-R^{(1)}\right) / R^{(1)}, \quad \eta=H / L, \quad \xi=a /\left(R^{(2)}-R^{(1)}\right) .
$$

Note that the radius of the outer shell $\left(R^{(2)}\right)$ is fixed, and the radius of the inner shell $\left(R^{(1)}\right)$ varies and depends on the parameter $k$.

\begin{tabular}{llll}
\hline $\begin{array}{l}\text { Geometric data } \\
\text { Parameter }\end{array}$ & Value & $\begin{array}{l}\text { Fluid properties } \\
\text { Parameter }\end{array}$ & Value \\
$\mathrm{L}, \mathrm{m}$ & 1 & $\rho_{f}, \mathrm{~kg} / \mathrm{m}^{3}$ & 1000 \\
$\mathrm{R}^{(2)}, \mathrm{m}$ & 0.1 & $c, \mathrm{~m} / \mathrm{s}$ & 1500 \\
$h^{(1)}=b^{(2)}=h, \mathrm{~m}$ & $5 \times 10^{-4}$ & & \\
\hline
\end{tabular}

Table 1: Geometrical parameters of the shells and physical properties of the fluid.

\begin{tabular}{llll}
\hline Parameter & Value & Parameter & Value \\
$c_{11}, \mathrm{GPa}$ & 126 & $e_{15}, \mathrm{C} / \mathrm{m}^{2}$ & 17 \\
$c_{12}, \mathrm{GPa}$ & 79.5 & $e_{24}, \mathrm{C} / \mathrm{m}^{2}$ & 17 \\
$c_{13}, \mathrm{GPa}$ & 84.1 & $e_{31}, \mathrm{C} / \mathrm{m}^{2}$ & -6.5 \\
$c_{22}, \mathrm{GPa}$ & 126 & $e_{32}, \mathrm{C} / \mathrm{m}^{2}$ & -6.5 \\
$c_{23}, \mathrm{GPa}$ & 84.1 & $e_{33}, \mathrm{C} / \mathrm{m}^{2}$ & 23.3 \\
$c_{33}, \mathrm{GPa}$ & 117 & $d_{11} \times 10^{-10}, \mathrm{~F} / \mathrm{m}$ & 150.3 \\
$c_{44}, \mathrm{GPa}$ & 23 & $d_{22} \times 10^{-10}, \mathrm{~F} / \mathrm{m}$ & 150.3 \\
$c_{55}, \mathrm{GPa}$ & 23 & $d_{33} \times 10^{-10}, \mathrm{~F} / \mathrm{m}$ & 130 \\
$c_{66}, \mathrm{GPa}$ & 23.3 & $\rho_{s}, \mathrm{~kg} / \mathrm{m}^{3}$ & 7500 \\
\hline
\end{tabular}

Table 2: Properties of piezoceramics PZT-5H.

\section{Verification of the numerical model}

The assessment of reliability of the constructed algorithm for the numerical investigation of an electroelastic thin-walled body was made by comparing its performance with the results of work [21]. Here the simply supported at both ends $(v=w=0)$ shell made of PZT-5H piezoceramics was investigated (Tab. 3). The boundary conditions for the fluid were specified as follows: $\phi=0$ at $x=0$ and $x=L$.

\begin{tabular}{llll}
\hline Geometric data & \multicolumn{3}{c}{ Fluid properties } \\
Parameter & Value & Parameter & Value \\
$\mathrm{L}, \mathrm{m}$ & 5 & $\rho_{f}, \mathrm{~kg} / \mathrm{m}^{3}$ & 1000 \\
$\mathrm{R}, \mathrm{m}$ & 1 & $c, \mathrm{~m} / \mathrm{s}$ & 1500 \\
$\mathrm{~h}, \mathrm{~m}$ & 0.02 & & \\
\hline
\end{tabular}

Table 3: Geometrical parameters of electroelastic shells and physical properties of the fluid [21].

A comparison between the natural frequencies of the empty shell and the shell filled with an ideal compressible fluid is given in Tab. 4. The number of half-waves in the circumferential and meridional directions is denoted by $j$ and $m$, respectively. According to the data presented in the table, there is good agreement between the results obtained by analytical and numerical solution of the problem.

The assessment of the reliability of the results obtained for coaxial shells $(\xi=0)$ made of isotropic elastic material and containing fluid in the gap between them was carried out by comparison with the results of analytical solution presented in [36]. In this case, the matrices $\mathbf{K}_{\psi}^{(i)}$ and $\mathbf{K}_{s \psi}^{(i)}$ in Eqn. (27) are excluded from the calculation, and the stiffness matrices 
$\tilde{\mathbf{D}}^{(i)}$ are generated in the known fashion using Young's modulus $E_{s}$ and Poisson's ratio $v$. Geometrical and physicomechanical parameters of the problem are summarized in Tab. 5, and a comparison of the obtained results is given in Tab. 6.

\begin{tabular}{|c|c|c|c|c|c|c|c|c|c|c|c|}
\hline \multicolumn{6}{|c|}{ Without fluid } & \multicolumn{6}{|c|}{ With fluid } \\
\hline \multicolumn{2}{|c|}{ Wave numbers } & \multicolumn{2}{|c|}{ Short circuited } & \multicolumn{2}{|c|}{ Open circuited } & \multicolumn{2}{|c|}{ Wave numbers } & \multicolumn{2}{|c|}{ Short circuited } & \multicolumn{2}{|c|}{ Open circuited } \\
\hline$j$ & $m$ & [21] & Present & [21] & Present & $j$ & $m$ & [21] & Present & [21] & Present \\
\hline \multirow[t]{5}{*}{1} & 1 & 88.2310 & 88.1261 & 93.7900 & 93.7266 & 1 & 1 & 44.7600 & 44.6100 & 48.2710 & 48.1062 \\
\hline & 2 & 216.260 & 217.239 & 228.227 & 229.386 & & 2 & 107.390 & 107.797 & 115.614 & 115.968 \\
\hline & 0 & 275.956 & 280.586 & 275.956 & 280.586 & & 3 & 154.131 & 154.925 & 166.065 & 166.705 \\
\hline & 3 & 311.647 & 313.207 & 330.649 & 332.755 & & 4 & 189.327 & 190.099 & 204.211 & 204.721 \\
\hline & 4 & 366.968 & 368.502 & 392.134 & 393.952 & & 5 & 217.190 & 217.515 & 234.469 & 234.471 \\
\hline \multirow[t]{5}{*}{2} & 1 & 37.3160 & 36.8135 & 40.1090 & 39.6043 & 2 & 1 & 19.9160 & 19.5781 & 21.4640 & 21.3157 \\
\hline & 2 & 114.210 & 114.107 & 122.677 & 122.616 & & 2 & 62.5970 & 62.3712 & 67.6230 & 67.4905 \\
\hline & 3 & 193.791 & 194.308 & 207.916 & 208.565 & & 3 & 108.575 & 108.650 & 117.248 & 117.433 \\
\hline & 4 & 259.346 & 260.087 & 278.434 & 279.316 & & 4 & 149.022 & 149.255 & 160.912 & 161.282 \\
\hline & 5 & 308.464 & 308.553 & 331.551 & 331.658 & & 5 & 182.817 & 182.814 & 197.400 & 197.590 \\
\hline
\end{tabular}

Table 4: A comparison of the natural vibration frequencies $\omega(\mathrm{Hz})$ of a simply supported shell with or without fluid at different combinations of electrical boundary conditions.

\begin{tabular}{llllll}
\hline $\begin{array}{l}\text { Geometric data } \\
\text { Parameter }\end{array}$ & Value & $\begin{array}{l}\text { Material properties } \\
\text { Parameter }\end{array}$ & Value & $\begin{array}{l}\text { Fluid properties } \\
\text { Parameter }\end{array}$ & Value \\
$L, \mathrm{~m}$ & 0.3 & $E_{s}^{(1)}=E_{s}^{(2)}=E_{s}, \mathrm{GPa}$ & 69 & $\rho_{f}, \mathrm{~km} / \mathrm{m}^{3}$ & 1000 \\
$\mathrm{R}^{(1)}, \mathrm{m}$ & 0.1 & $v^{(1)}=v^{(2)}=v$ & 0.3 & $c, \mathrm{~m} / \mathrm{s}$ & 1483 \\
$\mathrm{R}^{(2)}, \mathrm{m}$ & 0.15 & $\rho_{s}^{(1)}=\rho_{s}^{(2)}=\rho_{s}, \mathrm{~kg} / \mathrm{m}^{3}$ & 2700 & & \\
$h^{(1)}=b^{(2)}=h, \mathrm{~m}$ & 0.002 & & & & \\
\hline
\end{tabular}

Table 5: Physicomechanical and geometrical parameters of the system, consisting of elastic coaxial shells and a fluid.

\begin{tabular}{|c|c|c|c|c|c|c|c|c|c|}
\hline$j$ & $m$ & [36] & Phase mode & Present & $j$ & $m$ & [36] & Phase mode & Present \\
\hline \multirow[t]{7}{*}{1} & 1 & 391.1 & out-of-phase & 390.3 & 2 & 1 & 435.6 & out-of-phase & 434.9 \\
\hline & 2 & 846.7 & out-of-phase & 846.3 & & 2 & 907.1 & out-of-phase & 905.2 \\
\hline & 3 & 1397.5 & out-of-phase & 1396.5 & & 1 & 996.8 & in-phase & 995.2 \\
\hline & 1 & 1736.6 & in-phase & 1732.5 & & 3 & 1401.3 & out-of-phase & 1399.7 \\
\hline & 4 & 1908.5 & out-of-phase & 1907.3 & & $*$ & 1822.2 & mixed phase & 1819.1 \\
\hline & 5 & 2317.2 & out-of-phase & 2312.5 & & * & 1892.6 & mixed phase & 1891.2 \\
\hline & * & 2623.4 & mixed phase & 2579.5 & & $*$ & 2265.3 & mixed phase & 2262.2 \\
\hline \multirow[t]{7}{*}{3} & 1 & 403.0 & out-of-phase & 403.2 & 4 & 1 & 382.5 & out-of-phase & 383.3 \\
\hline & 1 & 671.3 & in-phase & 671.4 & & 1 & 561.9 & in-phase & 562.9 \\
\hline & 2 & 858.30 & out-of-phase & 857.7 & & 2 & 791.0 & out-of-phase & 792.0 \\
\hline & 2 & 1344.8 & in-phase & 1343.8 & & 2 & 1075.5 & in-phase & 1076.7 \\
\hline & 3 & 1352.4 & out-of-phase & 1351.5 & & 3 & 1267.5 & out-of-phase & 1268.8 \\
\hline & 4 & 1810.7 & out-of-phase & 1811.1 & & 3 & 1676.9 & in-phase & 1678.0 \\
\hline & 3 & 2010.6 & in-phase & 2008.6 & & 4 & 1729.2 & out-of-phase & 1731.3 \\
\hline
\end{tabular}

Table 6: A comparison of the natural vibration frequencies $\omega(\mathrm{Hz})$ of clamped-clamped coaxial shells containing an ideal compressible fluid in the annular gap between them.

Note that in [36], it was first shown that for two elastic coaxial shells, along with the in-phase (the direction and number of meridional half-waves $m$ coincide) and out-of-phase (opposite directions) vibration modes there also exist mixed modes (the number of half-waves varies). From the data presented in Tab. 6, we may conclude that the results obtained in the framework of the model used in this paper agree fairly well with the data of the analytical solution presented in [36]. 


\section{Natural frequencies of electroelastic shells}

Fig. 2 shows the graphs of the lowest natural frequencies of vibrations $\omega$ as a function of the dimensionless displacement of the inner shell $\xi$ at different filling levels $\eta$ for the gap $k=1 / 10$. The results were obtained for two configurations of electroelastic bodies under different boundary conditions. As is evident from the presented data, in the presence of eccentricity the frequencies decrease regardless of the choice of electrical and kinematic boundary conditions at the edges of the shells. According to Refs. [6, 7,9], the reason for such a behavior is the growth of the added fluid mass. In this case, a decrease in the level of a fluid and, consequently, a decrease in its the added mass contributes to a stronger dependence of the lowest vibration frequency on the axial misalignment of the shells and eventually leads to their greater relative decrease. The cantilevered structures are less sensitive to the eccentricity of the inner shell, so that over a fairly wide range of the parameter $\xi$ a decrease in the frequencies is inessential. Similar dependencies for other values of the annular gap $k$ do not demonstrate qualitative differences, which is supported by the data given in Tab. 7 .

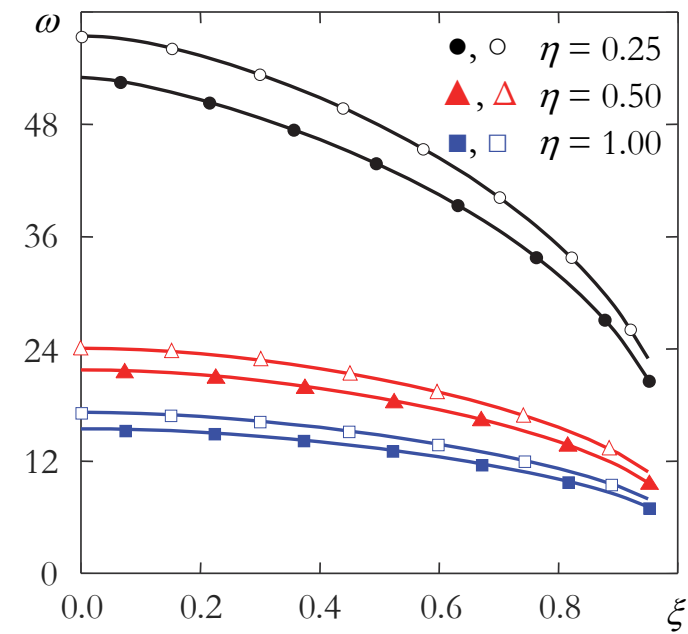

(a)

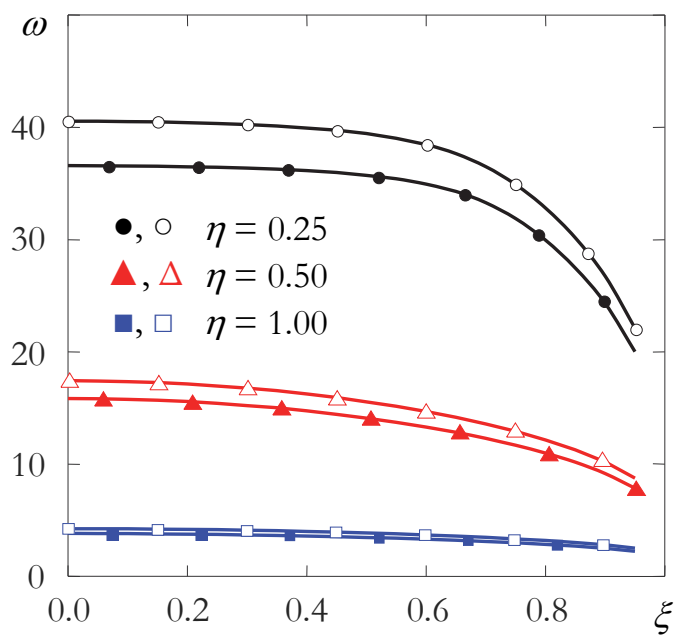

(b)

Figure 2: The dependences of the lowest vibration frequencies $\omega(\mathrm{Hz})$ on the eccentricity $\xi$ obtained at different filling levels and electrical boundary conditions (shaded symbols — short circuit mode, unshaded symbols — open circuit mode) for clamped-clamped (a) and cantilevered (b) shells, $k=1 / 10$

Due to an additional contribution of the associated stiffness, the electrical boundary conditions corresponding to the open-circuited configuration, lead to an increase in frequencies compared to the case of short-circuited configuration. The relative differences between these two variants $(\Delta \omega)$ as a function of eccentricity $\xi$ are presented in Figs. 3-5 for different values of the annular gap $k$. It can be seen from the above data that the curves are of a qualitatively different character, which is associated with the boundary conditions at the edges of the shells, the size of the annular gap and the level of filling with a liquid. The higher is the fluid level in the annular gap between the shells, the larger is the relative difference in frequencies $\Delta \omega$.

Fig. 6 shows the dependences, which allow us to estimate the variation in the lowest natural frequencies of vibrations with the level of the fluid in the gap at $k=1 / 10$ and various kinematic boundary conditions. The curves presented in the figure reflect the growth of frequencies with a decrease in the filling level. It can be seen that they are of qualitatively similar character for shells with different boundary conditions. However, in the case of rigid clamping at both ends (Fig. 6a), there is a range of the fluid levels, at which the frequencies practically do not change regardless of the displacement of the inner shell $\xi$. For cantilevered shells (Fig. 6b), such a behavior is not observed. In this case, the differences in the frequencies obtained at different electrical boundary conditions are insignificant.

Figs. 7, 8 show the mode shapes of the shells $(k=1 / 10$, open circuited, CC) obtained for different variants of the inner shell displacement and the fluid level in the annular gap. In the figures, displaying the transverse and longitudinal sections of the system, the dotted lines represent the shells in the undeformed state, and the solid line - the shells in the deformed state. The liquid is shown by light grey. 


\begin{tabular}{|c|c|c|c|c|c|c|c|c|}
\hline \multirow{2}{*}{$k$} & \multirow{2}{*}{$\eta$} & \multirow{2}{*}{$\xi$} & \multicolumn{3}{|c|}{ Clamped-Clamped shells } & \multicolumn{3}{|c|}{ Clamped-Free shells } \\
\hline & & & Short circuited & Open circuited & $\Delta \omega, \%$ & Short circuited & Open circuited & $\Delta \omega, \%$ \\
\hline \multirow[t]{9}{*}{$1 / 2$} & 1.00 & 0.00 & 32.7722 & 36.6289 & 11.7682 & 8.58148 & 9.65366 & 12.4942 \\
\hline & & 0.50 & 28.0167 & 30.9768 & 10.5657 & 7.78148 & 8.71879 & 12.0455 \\
\hline & & 0.95 & 15.9592 & 17.8405 & 11.7885 & 5.22212 & 5.88609 & 12.7145 \\
\hline & 0.50 & 0.00 & 44.0368 & 49.0209 & 11.3179 & 28.3814 & 31.4965 & 10.9758 \\
\hline & & 0.50 & 38.5264 & 42.7049 & 10.8459 & 26.7499 & 29.7033 & 11.0407 \\
\hline & & 0.95 & 21.5175 & 24.0634 & 11.8313 & 17.2344 & 19.2967 & 11.9662 \\
\hline & 0.25 & 0.00 & 89.2623 & 98.0454 & 9.83972 & 39.1212 & 43.3674 & 10.8539 \\
\hline & & 0.50 & 81.4270 & 90.0862 & 10.6343 & 39.0705 & 43.3060 & 10.8407 \\
\hline & & 0.95 & 45.0888 & 49.9111 & 10.6952 & 37.8442 & 41.8567 & 10.6027 \\
\hline \multirow[t]{9}{*}{$1 / 10$} & 1.00 & 0.00 & 15.4716 & 17.2113 & 11.2443 & 3.81831 & 4.25959 & 11.5569 \\
\hline & & 0.50 & 13.3385 & 14.7800 & 10.8069 & 3.47482 & 3.87567 & 11.5359 \\
\hline & & 0.95 & 7.13355 & 7.97309 & 11.7689 & 2.24936 & 2.52362 & 12.1930 \\
\hline & 0.50 & 0.00 & 21.7425 & 24.0692 & 10.7013 & 15.8505 & 17.4368 & 10.0074 \\
\hline & & 0.50 & 18.7429 & 20.7607 & 10.7661 & 14.1066 & 15.5642 & 10.3326 \\
\hline & & 0.95 & 9.70433 & 10.8434 & 11.7380 & 7.85436 & 8.76154 & 11.5500 \\
\hline & 0.25 & 0.00 & 53.0114 & 57.4618 & 8.39530 & 36.5821 & 40.5511 & 10.8496 \\
\hline & & 0.50 & 43.6699 & 47.9169 & 9.72512 & 35.7320 & 39.4371 & 10.3692 \\
\hline & & 0.95 & 20.7597 & 22.9591 & 10.5944 & 20.0218 & 22.0848 & 10.3036 \\
\hline \multirow[t]{6}{*}{$1 / 100$} & 1.00 & 0.00 & 4.97846 & 5.50687 & 10.6138 & 1.22342 & 1.35737 & 10.9483 \\
\hline & & 0.50 & 4.27499 & 4.72525 & 10.5323 & 1.11144 & 1.23392 & 11.0193 \\
\hline & & 0.95 & 2.25033 & 2.51305 & 11.6749 & 0.71178 & 0.79643 & 11.8917 \\
\hline & 0.50 & 0.00 & 7.08603 & 7.80343 & 10.1242 & 5.42042 & 5.92045 & 9.22498 \\
\hline & & 0.50 & 6.04697 & 6.68328 & 10.5228 & 4.69234 & 5.15888 & 9.94261 \\
\hline & & 0.95 & 3.06832 & 3.42595 & 11.6555 & 2.50227 & 2.78760 & 11.4030 \\
\hline
\end{tabular}

Table 7: Natural vibration frequencies $\omega(\mathrm{Hz})$ of the system of coaxial shells at different values of the annular gap $k$, eccentricity $\xi$, and filling level $\eta$ and varying electrical and kinematic boundary conditions.

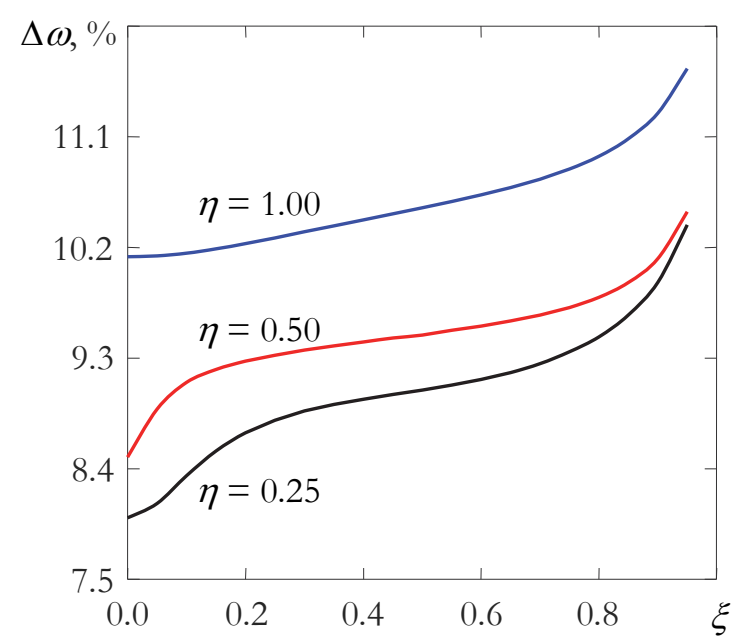

(a)

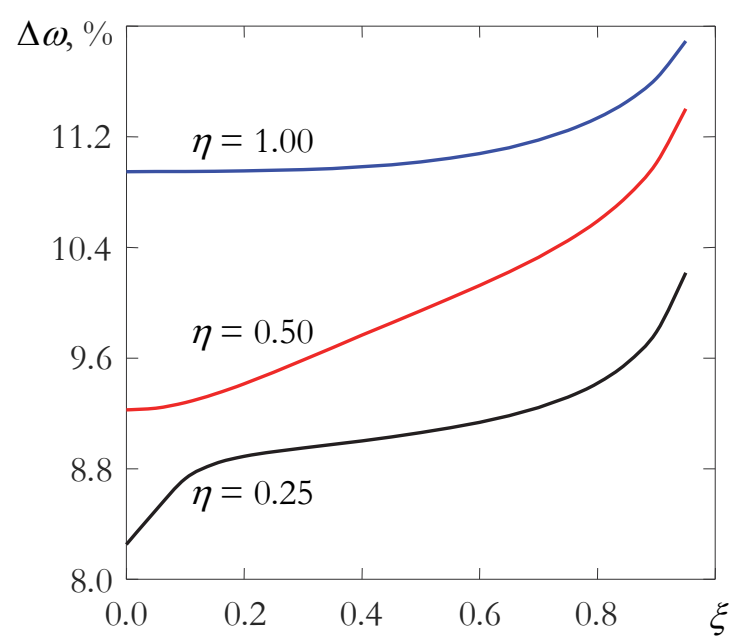

(b)

Figure 3: The relative difference in the frequencies $\Delta \omega$ as a function the eccentricity $\xi$ at different levels of the fluid in the annular gap $\eta$ for clamped-clamped (a) and cantilevered shells (b), $k=1 / 100$. 


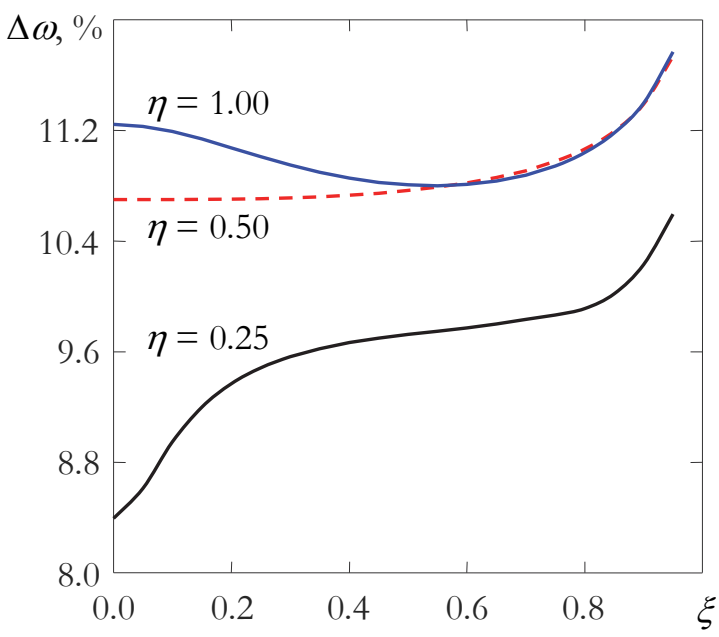

(a)

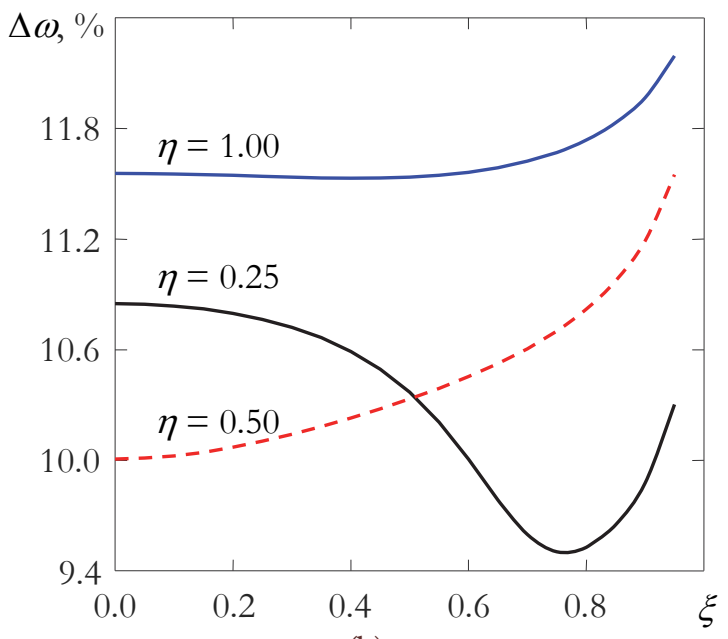

(b)

Figure 4: The relative difference in the frequencies $\Delta \omega$ as a function the eccentricity $\xi$ at different levels of the fluid in the annular gap $\eta$ for clamped-clamped (a) and cantilevered shells (b), $k=1 / 10$.

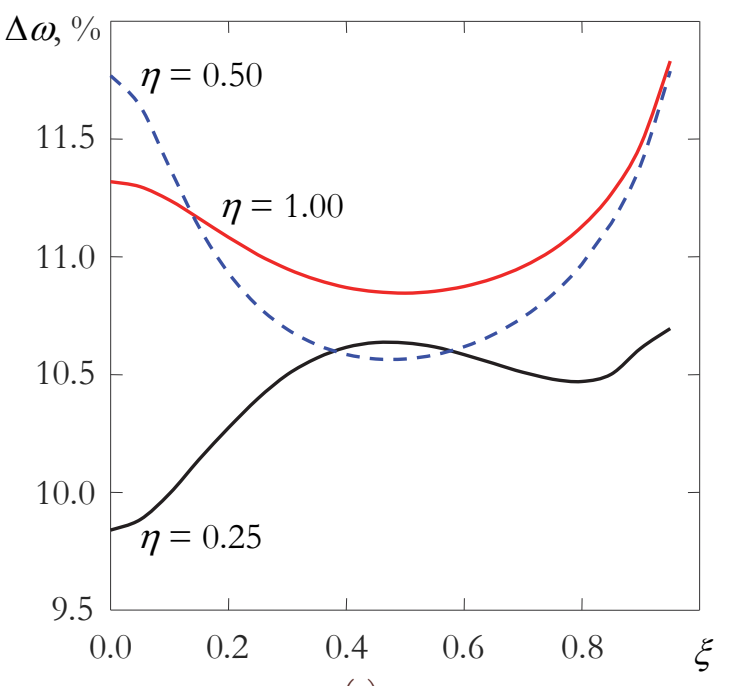

(a)

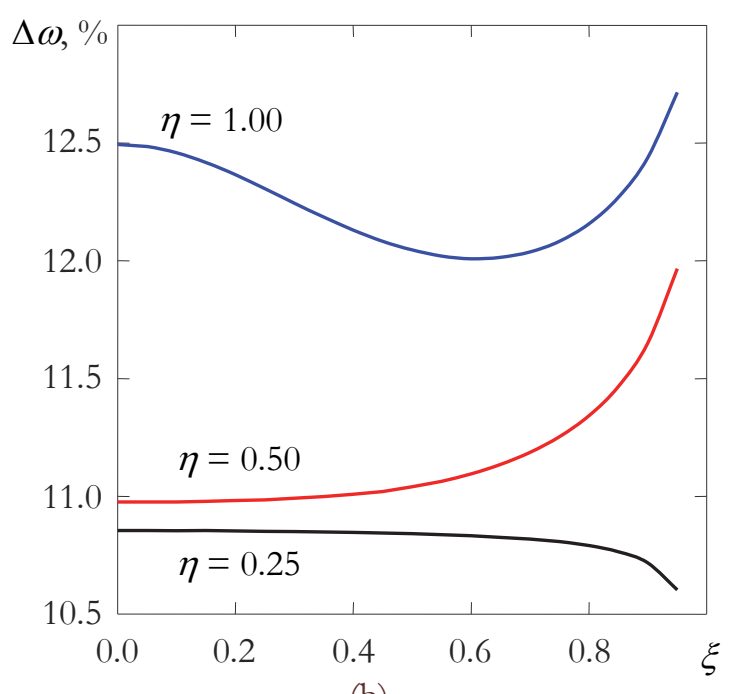

(b)

Figure 5: The relative difference in the frequencies $\Delta \omega$ as a function the eccentricity $\xi$ at different levels of the fluid in the annular gap $\eta$ for clamped-clamped (a) and cantilevered shells (b), $k=1 / 2$.

From the presented data it is clear that the amplitude of the displacements on the wetted areas of the shell surfaces exceeds the displacement amplitude on the areas not contacting with the fluid. A decrease in the level of the fluid also leads to a decrease in the maximum amplitudes of the displacements occurring in the shells. Moreover, in the case of the eccentricity, the displacement maximum occurs in the areas where the distance between the thin-walled bodies is minimal $\left(R^{(2)}-R^{(1)}-a\right)$ and gradually decreases towards the region where the spacing between them is maximum $\left(R^{(2)}-R^{(1)}+a\right)$. The behavior described above is the result of a change in the added mass of the fluid in the corresponding regions of the shells. 


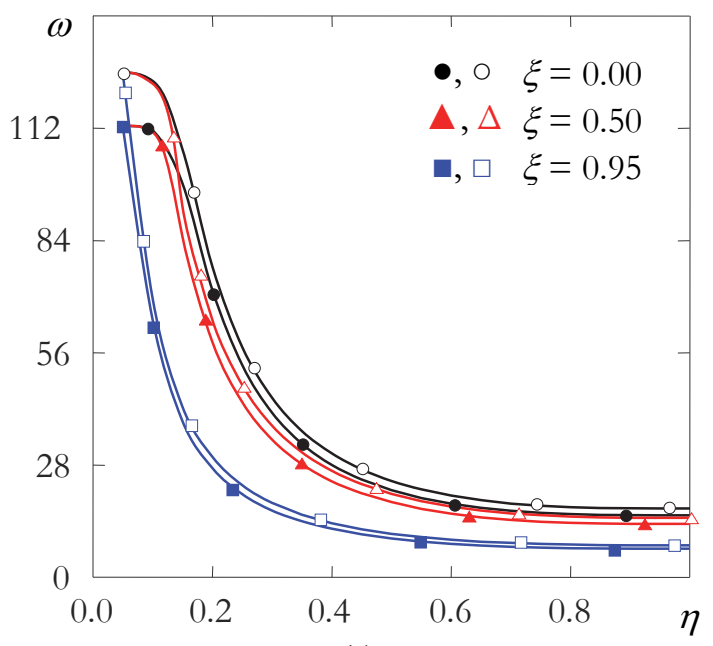

(a)

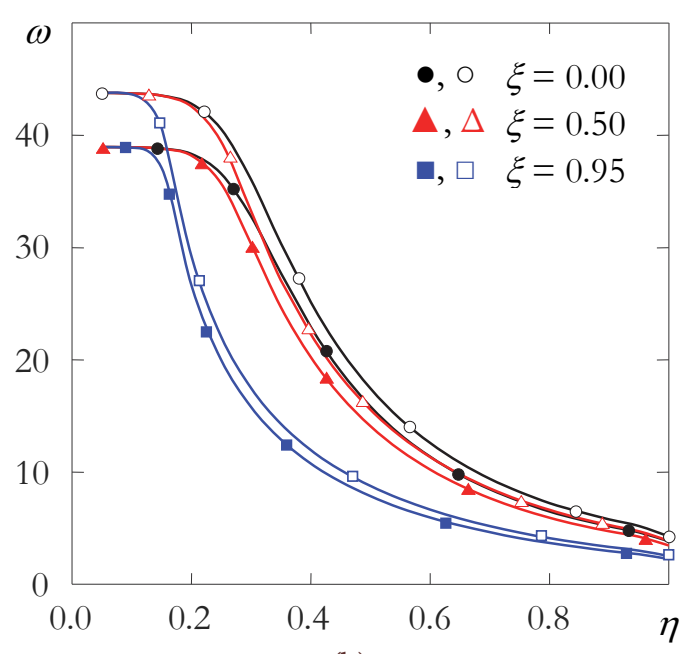

(b)

Figure 6: Variation in the lowest natural frequencies $\omega(\mathrm{Hz})$ with the level of the fluid in the annular gap between the shells $\eta$ at different values of the eccentricity $\xi$ and various electrical boundary conditions (shaded symbols — short-circuit mode, unshaded symbols — open-circuit mode) for clamped-clamped (a) and cantilevered (b) shells: $k=1 / 10$.

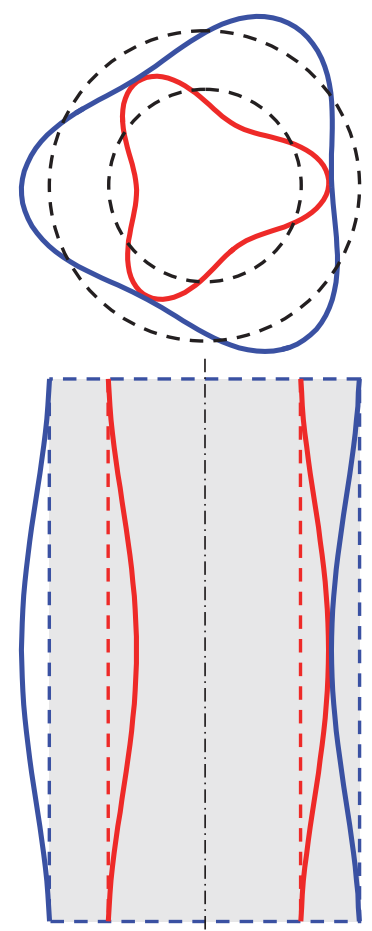

(a)
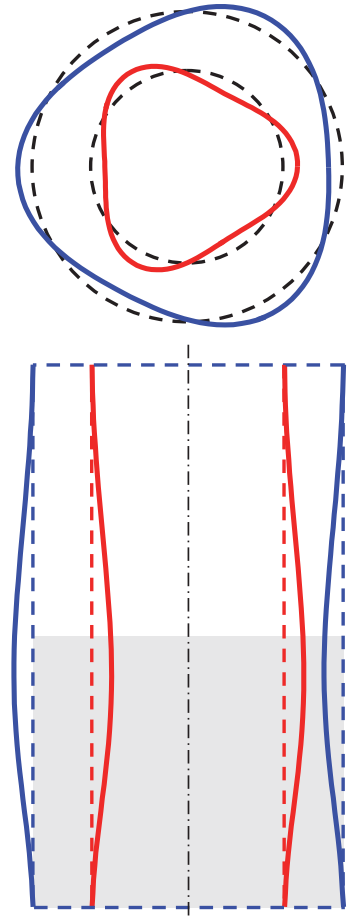

(b)
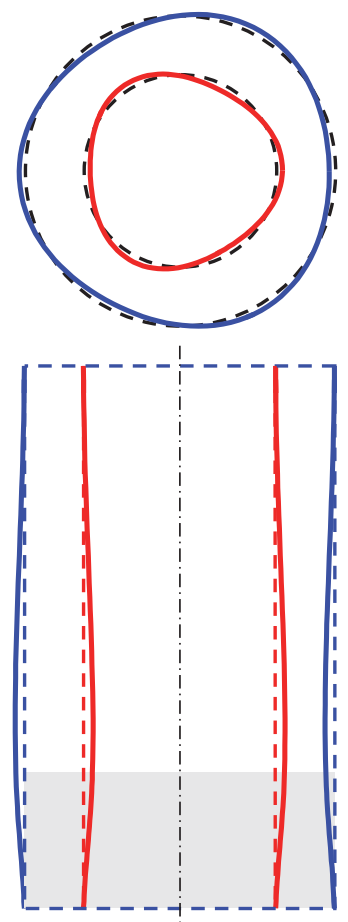

(c)

Figure 7: The lowest vibration mode of the electroelastic shell interacting with a fluid in the transverse $(x=L / 2)$ and longitudinal $(y=0)$ sections at different levels of the fluid in the annular gap $\eta$ (CC, open-circuit mode, $k=1 / 10, \xi=0):(a) \eta=1.0$; (b) $\eta=0.5 ;$ (c) $\eta=0.25$. 


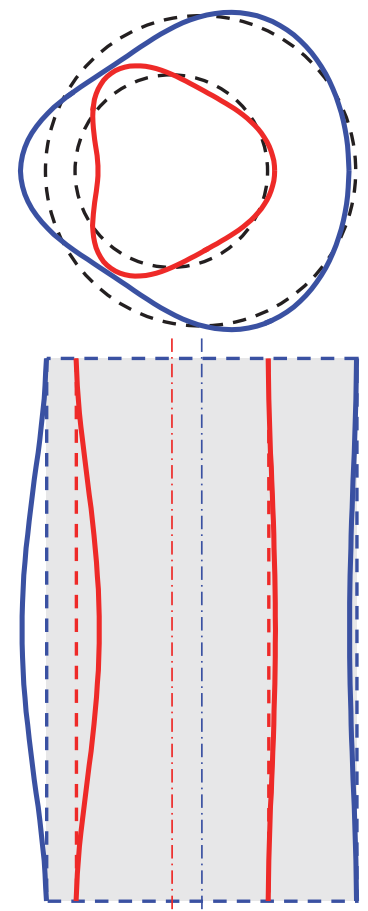

(a)

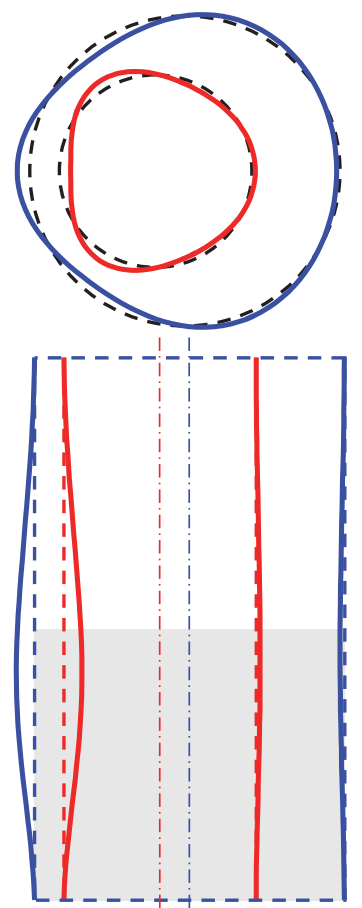

(b)
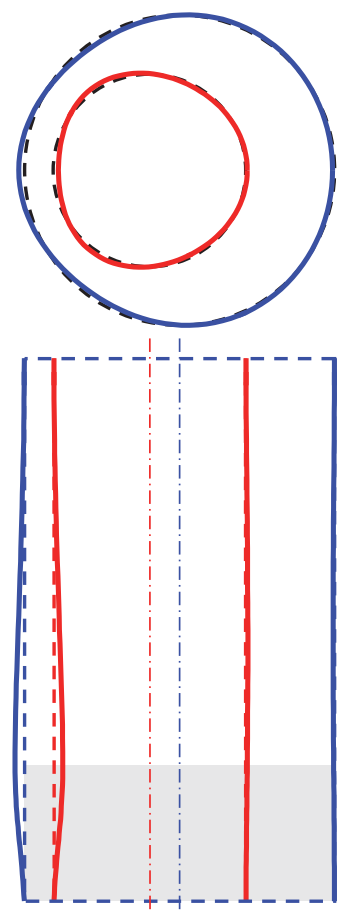

(c)

Figure 8: The lowest vibration mode of the electroelastic shell interacting with a fluid in the transverse $(x=L / 2)$ and longitudinal $(y=0)$ sections at different levels of the fluid in the annular gap $\eta$ (CC, open-circuit mode, $k=1 / 10, \xi=0.5):(a) \quad \eta=1.0$; (b) $\eta=0.5$; (c) $\eta=0.25$.

\section{CONCLUSION}

$\mathrm{T}$ he dynamic behavior of eccentric (non-coaxial) electroelastic cylindrical shells, containing a quiescent fluid in the annular gap between them, was studied in a three-dimensional formulation with the use of the proposed mathematical model, the numerical implementation of which was based on the finite element method. The analysis of the obtained results allowed us to estimate the influence of the size of the annular gap between the shell, the level of the fluid in it, the eccentricity of the inner shell, and the electrical and kinematic boundary conditions on the lowest natural frequencies of vibrations and the corresponding mode shapes. It was demonstrated that both an increase in the misalignment of the shells and the fluid level in the annular gap and a decrease in the size of the gap itself are responsible for a decay of the lowest vibration frequencies. The growth of the lowest frequencies is provided by setting on the electrode covered surfaces of the shells the electrical boundary condition peculiar to the open-circuited configuration.

\section{ACKNOWLEDGMENTS}

he study was supported by the grant of the Russian Scientific Foundation (project No. 18-71-10054).

\section{REFERENCES}

[1] Brown, S.J. (1982). A survey of studies into the hydrodynamic response of fluid-coupled circular cylinders, J. Press. Ves. Tech., 104(1), pp. 2-19. DOI: 10.1115/1.3264181 
[2] Païdoussis, M.P. (2016). Fluid-Structure Interactions: Slender Structures and Axial Flow, vol. 2, 2nd edn., London, Elsevier Academic Press.

[3] Bochkarev, S.A., Lekomtsev, S.V. and Senin, A.N. (2018). Analysis of spatial vibrations of coaxial cylindrical shells partially filled with a fluid, Comput. Continuum Mech., 11(4), pp. 448-462. DOI: 10.7242/1999-6691/2018.11.4.35

[4] Bochkarev, S.A., Lekomtsev, S.V., Matveenko, V.P. and Senin, A.N. (2019). Hydroelastic stability of partially filled coaxial cylindrical shells, Acta Mech. (in press)

[5] Buivol, B.N. and Guz, A.N. (1966). Oscillations of two cylindrical eccentrically arranged shells in a stream of inviscid liquid, Rep. AS Ukr. SSR, no. 11, pp. 1412-1415.

[6] Chung, H. and Chen, S.-S. (1977). Vibration of a group of circular cylinders in a confined fluid, J. Appl. Mech., 44(2), pp. 213-217. DOI: $10.1115 / 1.3424026$

[7] Mateescu, D., Païdoussis, M.P. and Sim, W.-G. (1994). Spectral solutions for unsteady annular flows between eccentric cylinders induced by transverse oscillations, J. Sound Vib., 177(5), pp. 635-649.

DOI: $10.1006 /$ jsvi.1994.1458.

[8] Jeong, K.-H. (1999). Dynamics of a concentrically or eccentrically submerged circular cylindrical shell in a fluid-filled container, J. Sound Vib., 224(4), pp. 709-732. DOI: 10.1006/jsvi.1999.2209.

[9] Jeong, K.-H., Lee G.-M. and Chang, M.-H. (2001). Free vibration analysis of a cylindrical shell eccentrically coupled with a fluid-filled vessel, Comput. Struct., 79(16), pp. 1517-1524. DOI: 10.1016/S0045-7949(01)00031-1.

[10] Baghdasaryan, G.Ye. (1968). The vibrations of coaxial cylindrical shells with a clearance partially filled with fluid, Mechanics. Proc. AS Arm. SSR, 21(4), pp. 40-47.

[11] Tani, J. and Haiji, H. (1986). The free vibration of free-clamped fluid-coupled coaxial cylindrical shells, Trans. Jpn. Soc. Mech. Eng. Part C, 52(484), pp. 3137-3144. DOI: 10.1299/kikaic.52.3137.

[12] Tani, J., Sakai, T. and Chiba, M. (1989). Dynamic stability of fluid-coupled coaxial cylindrical shells under vertical excitation, Trans. Jpn. Soc. Mech. Eng. Part C, 55(512), pp. 870-877. DOI: 10.1299/kikaic.55.870.

[13] Okazaki, K., Tani, J. and Sugano, M. (2002). Free vibrations of a laminated composite coaxial circular cylindrical shell partially filled with liquid, Trans. Jpn. Soc. Mech. Eng. Part C, 68(671), pp. 1942-1949. DOI: 10.1299/kikaic.68.1942.

[14] Gavrilova, E. (2003). A study of the vibration of fluid coupled coaxial cylindrical shell, In: Benaroya H., Wei T.J. (eds) IUTAM Symposium on Integrated Modeling of Fully Coupled Fluid Structure Interactions Using Analysis, Computations and Experiments. Fluid Mechanics and its Applications, 75, pp. 363-374.

DOI: 10.1007/978-94-007-0995-9_26.

[15] Baghdasaryan, G.Ye. and Marukhyan, S.A. (2011). Dynamic behavior of a coaxial cylindrical shells, with a gap partially filled with fluid, Mechanics. Proc. NAS Arm., 64(3), pp. 10-21.

[16] Ibrahim, R.A. (2005). Liquid Sloshing Dynamics: Theory and Applications, Cambridge, Cambridge University Press.

[17] Abramovich, H. (2016). Intelligent Materials and Structures, Berlin, De Gruyter.

[18] Kaljevic, I. and Saravanos, D.A. (1997). Steady-state response of acoustic cavities bounded by piezoelectric composite shell structures, J. Sound Vib., 204(3), pp. 459-476. DOI: 10.1006/jsvi.1996.0911

[19] Saravanos, D.A. (1997). Mixed laminate theory and finite element for smart piezoelectric composite shell structures, AIAA J., 35(8), pp. 1327-1333. DOI: 10.2514/2.264.

[20] Lammering, R. and Mesecke-Rischmann, S. (2003). Multi-field variational formulations and related finite elements for piezoelectric shells, Smart Mater. Struct., 12(6), pp. 904-913. DOI: 10.1088/0964-1726/12/6/007.

[21] Larbi, W., Deü, J.-F. and Ohayon, R. (2007). Vibration of axisymmetric composite piezoelectric shells coupled with internal fluid, Int. J. Num. Meth. Eng., 71(12), pp. 1412-1435. DOI: 10.1002/nme.1987.

[22] Deü, J.-F., Larbi, W. and Ohayon, R. (2008). Piezoelectric structural acoustic problems: Symmetric variational formulations and finite element results, Comp. Meth. Appl. Mech. Eng., 197(19-20), pp. 1715-1724.

DOI: $10.1016 /$ j.cma.2007.04.014.

[23] Larbi, W., Deü, J.-F. and Ohayon, R. (2012). Finite element formulation of smart piezoelectric composite plates coupled with acoustic fluid, Compos. Struct., 94(2), pp. 501-509. DOI: 10.1016/j.compstruct.2011.08.010.

[24] Bochkarev, S.A., Lekomtsev, S.V. and Matveenko, V.P. (2013). Numerical modeling of spatial vibrations of cylindrical shells, partially filled with fluid, Comput. technologies, 18(2), pp. 12-24.

[25] Bochkarev, S.A., Lekomtsev, S.V. and Matveenko, V.P. (2015). Natural vibrations of loaded noncircular cylindrical shells containing a quiescent fluid, Thin-Walled Struct., 90, pp. 12-22. DOI: 10.1016/j.tws.2015.01.001

[26] Bochkarev, S.A., Lekomtsev S.V. and Matveenko, V.P. (2016). Natural vibrations and stability of elliptical cylindrical shells containing fluid, Int. J. Struct. Stab. Dyn., 16(10), 1550076. DOI: 10.1142/S0219455415500765.

[27] Vol'mir, A.S. (1979). Shells in Liquid and Gas Flow. Hydroelastisity problems, Moscow, Nauka.

[28] Amabili, M. (1996). Free vibration of partially filled, horizontal cylindrical shells, J. Sound Vib., 191(5), pp. 757-780. 
DOI: $10.1006 /$ jsvi.1996.0154.

[29] Grinchenko, V.T., Ulitko, A.F. and Shul'ga, N.A. (1989). Mechanics of Coupled Fields in Elements of Structures. vol. 5. Electroelasticity, Kiev, Naukova Dumka.

[30] Rogacheva, N.N. (1994). The Theory of Piezoelectric Shells and Plates, London, CRC Press.

[31] Sheng, G.G. and Wang, X. (2010). Thermoelastic vibration and buckling analysis of functionally graded piezoelectric cylindrical shells, Appl. Math. Model., 34(9), pp. 2630-2643. DOI: 10.1016/j.apm.2009.11.024.

[32] Yao, G. and Li, F.-M. (2014). The stability analysis and active control of a composite laminated open cylindrical shell in subsonic airflow. J. Intel. Mat. Sys. Struct., 25(3), pp. 259-270. DOI: 10.1177/1045389X13491020.

[33] Zienkiewicz, O.C. (1971). Finite Element Method in Engineering Science, New York, McGraw-Hill.

[34] Reddy, J.N. (2015). An introduction to nonlinear finite element analysis, 2nd edn, Oxford, Oxford University Press.

[35] Lehoucq, R.B. and Sorensen, D.C. (1996). Deflation techniques for an implicitly restarted Arnoldi iteration, SIAM J. Matrix Anal. Appl., 17(4), pp. 789-821. DOI: 10.1137/S0895479895281484.

[36]Jeong, K.-H. (1998). Natural frequencies and mode shapes of two coaxial cylindrical shells coupled with bounded fluid, J. Sound Vib., 215(1), pp. 105-124. DOI: 10.1006/jsvi.1998.1648. 